\title{
« Traumatisme historique » et vote Front national : l'impact de la mémoire de la Guerre d'Algérie sur les opinions politiques des rapatriés
}

L'accession de Marine LE PEN au second tour de la présidentielle de 2017 témoigne de l'inscription profonde du Front National (FN), le parti d'extrême droite, dans le paysage politique français. Dans cet article, nous nous intéressons à un des électorats supposés de ce parti, celui des Français rapatriés d'Algérie, population communément appelée " pieds-noirs "'. Depuis les premiers suc-

L'auteure : Emmanuelle COMTAT est enseignantechercheuse LRU à l'Université Grenoble Alpes (département Information Communication) et chercheuse rattachée à I'UMR CNRS LARHRA (Laboratoire de Recherche Historique Rhône-Alpes). cès électoraux du FN dans les années 1980, le vote des pieds-noirs en faveur de ce parti a régulièrement été évoqué par les médias. Vote $\mathrm{FN}$ et "vote pied-noir " ont souvent été confondus, comme si cette " communauté » était entièrement acquise à l'extrême droite. À la veille d'échéances électorales importantes, les rapatriés continuent de susciter l'intérêt des hommes politiques ${ }^{2}$, convaincus que leur vote peut encore être déterminant, au moment où les résultats des scrutins risquent d'être serrés face au FN. La déclaration à Toulon d'Emmanuel MACRON aux rapatriés, "Je vous ai compris et je vous aime", avant le premier tour de la présidentielle de 2017 pour éteindre la polémique suscitée lors de sa visite à Alger où il avait qualifié la colonisation de "crime contre l'Humanité » montre que le passé colonial demeure sensible et s'immisce dans les débats publics et dans la campagne électorale. Nous proposons ici d'examiner l'ampleur du vote $\mathrm{FN}$ au sein de ce groupe et les caractéristiques des pieds-noirs qui votent pour ce parti.

Les études de références en science politique mettent en évidence que la filière "rapatriés " est l'une des composantes de l'extrême droite française. Pendant la Guerre d'Algérie, le nationalisme faisait corps avec la défense de l'empire colonial ${ }^{3}$. Par ailleurs, au premier tour de la présidentielle de 1965, les rapatriés furent vraisembla-

\footnotetext{
1 Les pieds-noirs sont les Français (venus de France métropolitaine, d'États européens ou juifs sépharades d'Algérie) installés en Algérie de 1830 à 1962.

2 IFOP, "Le vote pied-noir : mythe ou réalité », Focus, 107, 2014, p. 1.

3 Bon Frédéric, CheYLAN Jean-Paul, La France qui vote, Paris, Hachette, 1988, p. 256.
} 
blement nombreux à soutenir le candidat de l'extrême droite TIXIERVIGNANCOUR pour sanctionner DE GAULLE de sa politique algérienne ${ }^{4}$. Dans les cadres fondateurs du FN en 1972, on retrouve des anciens de l'OAS, l'organisation terroriste pro Algérie française ${ }^{5}$. Lors de la première percée électorale du FN aux élections européennes de 1984, ses zones de force (région lyonnaise, île-de-France, Nord-Est, littoral méditerranéen, sillon rhodanien) coïncident avec la localisation de l'industrie française et de l'immigration algérienne ${ }^{6}$. Son bastion méridional correspond aux régions d'implantation de pieds-noirs. Dans des villes du Midi (Toulon, etc.), des réseaux se constituent alors entre des amicales de rapatriés et ce parti. Des pieds-noirs figurent également parmi les candidats du FN dans ces régions. Toutefois, s'il conserve son ancrage méditerranéen, le $\mathrm{FN}$ a progressé, ces vingt dernières années, dans le Nord et l'Est, régions jadis industrielles, aujourd'hui en voie de désindustrialisation ${ }^{8}$. Or ces territoires ne sont pas ceux où les rapatriés ont été nombreux à se réinstaller en $1962^{\circ}$. Le vote frontiste s'est ainsi diffusé dans cette France périphérique et périurbaine fragilisée par la mondialisation, et dans l'électorat ouvrier votant autrefois à gauche et dans celui des petits commerçants et artisans ${ }^{10}$. Ces travaux ont donc mis en évidence que certains pieds-noirs soutiennent le FN, mais que le vote pour ce parti n'est pas uniquement le fait des rapatriés, comme l'attestent l'élection présidentielle de 2002 (JeanMarie LE PEN, leader du FN, obtenait 4,8 millions de voix au premier tour et accédait au second tour où il remportait 5,5 millions de voix) et l'élection présidentielle de 2017 (où Marine LE PEN obtenait successivement 7,6 puis 10,6 millions de suffrages exprimés au premier et au second tour)", d'autant qu'il s'agit d'une population vieillissante dont le poids électoral est moindre que par le passé.

\footnotetext{
4 Goguel François, Chroniques électorales. La cinquième république du général de Gaulle, Paris, FNSP, 1982, pp. 383-393.

5 Camus Jean-Yves, "Origine et formation du Front national », in MAYER Nonna et Perrineau Pascal (dir.), Le Front national à découvert, Paris, FNSP, 1996, pp. 17-19.

6 Bon Frédéric, CheYLAN Jean-Paul, La France qui vote, op. cit., p. 234 et StORA Benjamin, Le transfert d'une mémoire. De l'Algérie française au racisme anti-arabe, Paris, La Découverte, 1999, p. 95.

7 VEUgELERS John, «Ex-Colonials, Voluntary Associations, and Electoral Support for the Contemporary Far Right ", Comparative European Politics, 3, 2005, pp. 408-431 et VEUGELERS John, "Colonial past, voluntary associations, and far-right voting in France", Ethnic and Racial Studies Review, 38, 5, 2015, pp. 775-791.

8 GOMBIN Joël, "Le changement dans la continuité. Géographie électorale du FN depuis 1992 ", CRÉPON Sylvain, DÉzé Alexandre et MAYER Nonna (dir.), Les faux-semblants du Front national. Sociologie d'un parti politique, Paris, Presses de Sciences Po, 2015, pp. 395-416.

9 BON Frédéric, CHEYLAN Jean-Paul, La France qui vote, op. cit., p. 245.

10 Perrineau Pascal, “Les étapes d'une implantation électorale », Mayer Nonna et Perrineau Pascal (dir.), Le Front national à découvert, op. cit., pp. 37-62 et Perrineau Pascal, La France au Front, Paris, Fayard, 2014, pp. 34-35 et pp. 107-159.
} 
Pour évaluer l'impact de la mémoire traumatique sur le vote, on réinterroge les travaux de l'historien Paul Boıs sur le modèle du «traumatisme historique " qui met en exergue l'importance du contexte historique et social dans le façonnement des comportements électoraux ${ }^{12}$. La mémoire de la Guerre d'Algérie (1954-1962) est chargée d'éléments traumatiques ${ }^{13}$ susceptibles d'agir sur les orientations partisanes des pieds-noirs. Dans quelle mesure ce passé traumatique influe-t-il sur le positionnement à l'extrême-droite d'une partie des rapatriés et de leurs descendants? Le modèle du "traumatisme historique » vient-il, dans ce groupe, concurrencer les variables sociodémographiques ordinairement explicatives du vote FN ? Les motivations des pieds-noirs à voter pour l'extrême droite diffèrent-elles de celles des autres Français qui votent pour le FN?

Nous analysons, dans une première partie, les logiques qui conduisent certains pieds-noirs à voter pour le $\mathrm{FN}$ puis, dans une seconde partie, les caractéristiques sociodémographiques des rapatriés votant pour ce parti.

Pour donner une estimation du vote $\mathrm{FN}^{14}$ des pieds-noirs et des descendants, nous nous appuyons sur les résultats d'une enquête qualitative à partir d'entretiens semi directifs ${ }^{15}$ et sur deux enquêtes quantitatives sur le vote de ce groupe (l'enquête pied-noir 2002 effectuée par nos soins ${ }^{16}$ et l'enquête préélectorale de 2012 de l'IFOP et du CEVIPOF sur les intentions de vote des rapatriés ${ }^{17}$ ), réalisées à dix ans d'intervalle, aux élections présidentielles de 2002 et de 2012.

\footnotetext{
12 Bois Paul, Paysans de l'Ouest, Paris, Flammarion, 1971 et Mayer Nonna et Perrineau Pascal (dir.), Les comportements politiques, Paris, A. Colin, 1992, pp. 44-46.

13 Notamment le fait d'avoir vécu la Guerre d'Algérie au quotidien, le terrorisme, les enlèvements de civils, la perte du territoire et des biens matériels, le mauvais accueil ressenti à leur arrivée en métropole, le déracinement, le sentiment que leur mémoire n'est pas reconnue.

14 On ne peut connaître aujourd'hui avec exactitude le vote des pieds-noirs, puisque ils se sont mêlés après 1962 au reste du corps électoral. Nous devons donc recourir à des enquêtes d'opinion sur le vote de cette population.

1580 entretiens ont été réalisés par nos soins de 2000 à 2016 auprès de rapatriés et de descendants. 16 L'enquête pieds-noirs 2002 (UMR PACTE CNRS) a été menée auprès de 6000 rapatriés nés en Algérie avant 1962 et domiciliés dans les Alpes-Maritimes, l'Hérault et l'Isère. Elle a été réalisée quarante ans après le rapatriement de 1962 et six mois après le séisme politique du 21 avril 2002 où J.-M. LE PEN accéda au second tour de la présidentielle. Le questionnaire porte sur les comportements politiques en Algérie et en France depuis 1962; dans CомтAт Emmanuelle, Les pieds-noirs et la politique, Paris, Presses de Sciences Po, 2009. 17 L'enquête comporte plusieurs vagues durant la campagne électorale; SCIENCES PO, CEVIPOF, IFOP, « Le vote pied-noir 50 ans après les accords d'Évian, Élections 2012 ", Les élections sociologiques, 6, 2012 et IFOP, 


\section{Les logiques du vote FN dans le groupe des rapatriés}

Tableau $n^{\circ} 1$ Intentions de vote au $1^{\text {er }}$ et au $2^{\text {ème }}$ tour de la présidentielle de 2012

\begin{tabular}{|c|c|c|c|c|c|}
\hline & Pieds-noirs & $\begin{array}{l}\text { Non pieds- } \\
\text { noirs } 50 \text { ans } \\
\text { et }+\end{array}$ & $\begin{array}{l}\text { Descendants } \\
\text { de pieds-noirs }\end{array}$ & $\begin{array}{l}\text { Personnes } \\
\text { sans ascen- } \\
\text { dance pied- } \\
\text { noir }\end{array}$ & $\begin{array}{l}\text { Ensemble } \\
\text { des inscrits }\end{array}$ \\
\hline \multicolumn{6}{|l|}{$\begin{array}{l}\underline{1}^{\text {er }} \text { tour (sondage octobre } \\
2 \mathrm{O} 11 \text { ) }\end{array}$} \\
\hline Fr. HOLLANDE (PS) & $26 \%$ & - & $31 \%$ & - & $29 \%$ \\
\hline Centristes & $9 \%$ & - & $14 \%$ & - & $15,5 \%$ \\
\hline N. SARKOZY (UMP) & $26 \%$ & - & $15 \%$ & - & $22,5 \%$ \\
\hline M. Le Pen (FN) & $28 \%$ & - & $24 \%$ & - & $19,5 \%$ \\
\hline \multicolumn{6}{|l|}{$\begin{array}{l}\text { 1er tour (sondages cumu- } \\
\text { lés janvier-avril 2012) }\end{array}$} \\
\hline $\begin{array}{l}\text { Extrême gauche + J.-L. } \\
\text { MÉLENCHON }\end{array}$ & $5 \%$ & $12,5 \%$ & $13,5 \%$ & $13 \%$ & $13 \%$ \\
\hline $\begin{array}{l}\text { Fr. Hollande (PS) + Eva } \\
\text { Joly (Verts) }\end{array}$ & $\begin{array}{l}23,5 \% \\
(22 \%+1,5 \%)\end{array}$ & $\begin{array}{l}31 \% \\
(29,5 \%+1,5 \%)\end{array}$ & $\begin{array}{l}28 \% \\
(25 \%+3 \%)\end{array}$ & $\begin{array}{l}31 \% \\
(28,5 \%+2,5 \%)\end{array}$ & $\begin{array}{l}30,5 \% \\
(28 \%+2,5 \%)\end{array}$ \\
\hline $\begin{array}{l}\text { Fr. BAYROU (centriste } \\
\text { Modem) }\end{array}$ & $7 \%$ & $9,5 \%$ & $9 \%$ & $9 \%$ & $9 \%$ \\
\hline $\begin{array}{l}\text { N. SARKOZY (UMP) + } \\
\text { Dupont-Aignan }\end{array}$ & $\begin{array}{l}42 \% \\
(38 \%+4 \%) \\
\end{array}$ & $\begin{array}{l}32 \% \\
(30,5 \%+1,5) \\
\end{array}$ & $\begin{array}{l}29 \% \\
(26 \%+3 \%)\end{array}$ & $\begin{array}{l}29 \% \\
(27 \%+2 \%)\end{array}$ & $\begin{array}{l}29,5 \% \\
(27,5 \%+2 \%)\end{array}$ \\
\hline M. LE Pen (FN) & $21,5 \%$ & $14 \%$ & $20 \%$ & $18 \%$ & $18 \%$ \\
\hline \multicolumn{6}{|l|}{$\begin{array}{l}2^{\text {ème }} \text { tour (sondage cumu- } \\
\text { lés janvier-avril 2012) }\end{array}$} \\
\hline Fr. HOLLANDE & $36 \%$ & $51,5 \%$ & $48 \%$ & $52 \%$ & - \\
\hline N. SARKOZY & $64 \%$ & $48,5 \%$ & $52 \%$ & $48 \%$ & - \\
\hline
\end{tabular}

Sources: IFOP/CEVIPOF 201218 et IFOP 201219

Nos différents matériaux empiriques montrent que le vote des rapatriés est très hétérogène (tableau $n^{\circ} 1$ ). Néanmoins, les piedsnoirs sont majoritairement de droite et sont relativement nombreux à avoir déjà voté $\mathrm{FN}^{20}$. Dans l'enquête pied-noir 2002, 44\% des rapatriés sondés et $29 \%$ des descendants déclarent avoir voté au moins une fois pour ce parti depuis sa création en $1972^{21}$. A la présidentielle de 2002, une enquête IFOP «sortie des urnes » montre que 30\% des

18 Sciences Po, CEVIPOF, IFOP, «Le vote pied-noir 50 ans après les accords d'Evian, Élections 2012 », op.

cit., p. 2.

19 IFOP, « Le vote pied-noir : mythe ou réalité », op. cit., pp. 4-6.

20 CомтAт Emmanuelle, Les pieds-noirs et la politique, op. cit., pp. 237-267.

21 La question n'a jamais été posée en ces termes au reste des Français. On ne sait combien de personnes parmi eux ont voté au moins une fois pour ce parti. L'approche est ici expérimentale. 
pieds-noirs ont alors voté au premier tour pour Jean-Marie LE PEN ou pour Bruno MÉGRET ${ }^{22}$, soit un écart supérieur de 10 points par rapport à l'ensemble des Français ${ }^{23}$. L'enquête préélectorale du CEVIPOF et de l'IFOP (tableau $n^{\circ} 1$ ) sur les intentions de vote à la présidentielle de 2012 (sondage d'octobre 2011) indique que 28\% des pieds-noirs envisagent de voter pour Marine LE PEN (24\% des descendants) contre $26 \%$ des pieds-noirs pour Nicolas SARKOZY (15\% des descendants) ${ }^{24}$. Quelles que soient les élections, un sur-vote en faveur de la droite et de l'extrême-droite subsiste dans ce groupe ${ }^{25}$. L'électorat pied-noir montre ainsi des affinités avec le FN, même si le vote pour ce parti est loin d'être majoritaire chez les rapatriés.

Dans les entretiens, des pieds-noirs disent souvent ouvertement qu'ils sont électeurs du FN comme si aucun tabou ne pesait sur ce vote. C'est en soi une spécificité. Une «banalisation » du vote FN se fait jour dans une partie de l'électorat rapatrié. Elle semble s'être opérée assez tôt - avant même que Marine LE PEN ait adopté une stratégie de "dédiabolisation " pour attirer de nouveaux électeurs - comme le suggèrent des entretiens réalisés dans les années 2000 lorsque des déclarations de Jean-Marie LE PEN, son leader de l'époque, avaient marginalisé ce parti. Le $\mathrm{FN}$ apparaît à certains rapatriés comme un parti de droite comme un autre.

Toutefois, des rapatriés, comme d'autres Français, ont sans doute voté tardivement pour le FN. De 1965 à 1983, l'extrême droite connaît une traversée du désert, obtenant aux élections des scores limités ${ }^{26}$. Dans les départements à forte implantation de rapatriés, les résultats électoraux du FN demeurent modestes ${ }^{27}$. Dès 1984, l'extrême droite reprend de l'importance aux élections européennes. Comme leurs compatriotes, des pieds-noirs ont probablement commencé à voter

\footnotetext{
22 Après avoir pris ses distances avec le FN, Bruno. MÉGRET, qui souhaitait mener une stratégie d'alliances avec la droite parlementaire, fonde en 1999 le Mouvement National Républicain (MNR), parti d'extrême droite concurrent du FN.

23 IFOP, "Le vote pied-noir : mythe ou réalité », op. cit.

24 SCIENCES PO, CEVIPOF, IFOP, "Le vote pied-noir 50 ans après les accords d'Évian, Élections 2012 ", op. cit., pp. 2-3.

25 IFOP, «Le vote pied-noir : mythe ou réalité », op. cit., p. 5.

26Perrineau Pascal, La France au Front, op. cit., pp. 17-18.

27 À la présidentielle de 1974, J.-M. LE PEN obtient au premier tour 1,22\% des suffrages exprimés dans les Alpes-Maritimes, 1,13\% dans les Bouches-du-Rhône, 1,32\% dans l'Hérault, 1,30\% dans le Var et 1,19\% dans le Vaucluse. Dans ces départements, les scores du FN restent faibles même s'ils sont supérieurs à la moyenne nationale (0,75\% des voix). Aux législatives de 1978, le $\mathrm{FN}$ a au premier tour 1,18\% des suffrages dans les Alpes-Maritimes, 1,07\% dans les Bouches-du-Rhône et 0,96\% dans l'Hérault (contre 0,8\% des voix au plan national). Aux législatives de 1981, l'extrême droite a 0,88\% des voix au premier tour dans les AlpesMaritimes, 1,29\% dans les Bouches-du-Rhône, 0,98\% dans l'Hérault (0,5\% au plan national), dans COMTAT 
davantage pour le FN à ce moment là. Aux Européennes de 1984, les candidats du FN obtiennent $15,3 \%$ des suffrages exprimés dans l'Hérault, 19,5\% dans les Bouches-du-Rhône et 21,4\% dans les AlpesMaritimes. La percée du FN résulte alors de la montée du chômage après les chocs pétroliers et s'articule autour des enjeux de l'immigration. L'arrivée de la gauche au pouvoir en 1981 entraîne une radicalisation d'une partie de l'électorat de droite ${ }^{28}$. L'alternance a pu aussi exacerber les tensions chez des rapatriés qui ont pu penser que la gauche leur préférait les immigrés, renvoyant aussi à d'anciens clivages lors de la Guerre d'Algérie sur la question du maintien de l'Empire ou de la décolonisation.

Plusieurs logiques sont à l'œuvre chez les rapatriés électeurs du FN qui font référence à la mémoire de ce groupe et à des problèmes sociopolitiques récents (chômage, insécurité, terrorisme).

\section{Une dimension de reconnaissance}

Le FN n'existait pas au moment de la Guerre d'Algérie. Mais, JeanMarie LE PEN, député poujadiste ${ }^{29}$ de 1956 à 1958, a été l'un des rares hommes politiques à avoir alors publiquement soutenu les Français d'Algérie. L'extrême droite resurgit au premier plan lorsque DE GAULLE annonce en 1959 son choix en faveur de l'autodétermination. JeanMarie LE PEN est de ceux qui vont lutter pour le maintien de l'Algérie française et pour balayer la Ve République, afin de mettre en place un "ordre nouveau " $^{30}$. II s'engage pour aller combattre en Algérie. Dans les entretiens, des pieds-noirs rappellent que le leader du FN a pris fait et cause en leur faveur tandis qu'ils se sont sentis trahis et abandonnés par le reste de la classe politique française qui promettait de conserver l'Algérie et qui a finalement accepté son indépendance. De plus, à la création du FN en 1972, le parti a orienté son discours sur la "nostalgie " de l'Empire ${ }^{31}$. Jean-Marie LE PEN a aussi toujours exprimé de la sympathie à l'égard des rapatriés alors que leur persistance à soutenir le passé colonial les marginalisait dans l'opinion publique. Des pieds-noirs lui en furent reconnaissants et n'hésitèrent pas en retour à voter pour le FN. Des rapatriés ont aussi le sentiment que leurs revendications de groupe (reconnaissance mémorielle, etc.)

\footnotetext{
28 Perrineau Pascal, La France au Front, op. cit., pp. 63.

29 Le poujadisme (son fondateur, Pierre POUJADE) est un mouvement politique français d'extrême droite qui défendait dans les années 1950 les intérêts des commerçants et des artisans.

30 Perrineau Pascal, Le symptôme Le Pen. Radiographie des électeurs du Front national, Paris, Fayard,

1997, pp. 15-16.
}

31 Bon Frédéric, CheYLAN Jean-Paul, La France qui vote, op. cit., p. 256. 
sont mieux défendues par ce parti. Aujourd'hui, Marine LE PEN continue d'envoyer des messages aux rapatriés. Mais soucieuse d'élargir sa base électorale, elle a adopté un discours qui s'adresse moins qu'avant à ceux qui se sentent les victimes et oubliés de l'Histoire qu'à ceux de la mondialisation. Toutefois, des élus ou candidats FN du Midi continuent de tenir compte de l'électorat rapatrié et n'oublient pas de lui adresser des "gages » de loyauté en faisant référence à sa mémoire ou en menant des politiques publiques mémorielles en sa faveur. Robert MÉNARD, descendant de pied-noir et élu maire de Béziers en 2014 avec le soutien du FN, milite ainsi pour la suppression de la date du 19 mars pour commémorer la Guerre d'Algérie, revendication chère aux associations de rapatriés ${ }^{32}$. Marion Marechal-LE PEN, petite-fille de Jean-Marie LE PEN, a adressé des messages de sollicitude aux rapatriés. II en est de même, aux législatives de 2017, du candidat FN Louis ALIOT, fils d'une pied-noir et compagnon de Marine LE PEN, qui a été élu député des Pyrénées-Orientales où résident un nombre important de rapatriés.

\section{Une dimension anti immigrés en raison de la Guerre d'Algérie}

Les pieds-noirs qui votent pour le $\mathrm{FN}$ ne soutiennent généralement pas toutes les thèses de ce parti. Le thème de l'immigration, central dans le discours politique du FN, prime dans leur positionnement. Cet électorat adhère ordinairement aux propositions du FN de préférence nationale en matière sociale et familiale, de rejet de l'immigration et de fermeture des frontières.

\footnotetext{
LE PEN veut mettre les Arabes dehors, ben, il a raison! On est en France ou on n'est pas en France? Qu'il fasse d'abord aux Français et après on verra les étrangers! II a raison parce qu'il y en a marre ! (homme, 73 ans, pompier à la retraite, Algérois, Hérault).

On a du chômage, il faut donner du travail aux Français d'abord [...] On en a plus que jamais (les Algériens) et ils viennent foutre la pagaille, parce que toute la pagaille vient d'eux, c'est ça qui nous écœure et qui fait voter LE PEN! (homme, 67 ans, retraité du secteur privé, Oranien, Isère).
}

\footnotetext{
32 Le 19 mars 1962 est le jour d'entrée en vigueur du cessez-le-feu de la Guerre d'Algérie après la signature des accords d'Évian. Des associations de rapatriés et d'anciens combattants (UNC, Souvenirs français) s'opposent à cette date de commémoration au profit du 5 décembre (date officielle de 2003 à 2012), prétextant qu'on ne célèbre pas une défaite et qu'il y a eu de nombreuses victimes après les accords d'Évian. Le choix d'une date commémorative épouse le clivage gauche-droite. En 2012, le Parlement à majorité de 
Le FN a essayé de capter l'électorat des rapatriés en jouant sur sa rancœur. Benjamin STORA considère qu'il a puisé dans le vivier des "mémoires blessées " par l'humiliation de 1962 et qu'il a encouragé une "mémoire de la revanche " dirigée contre les immigrés maghrébins $^{33}$. Pascal PERRINEAU indique que la vision frontiste de l'immigration de Jean-Marie LE PEN ciblait avant tout les immigrés nord-africains et stigmatisait les filles et fils «ingrats " dont les pères avaient rejeté le lien avec la «France coloniale généreuse " ${ }^{34}$. Dans nos enquêtes, le vote de rapatriés pour le $\mathrm{FN}$ est directement lié à la perte de l'Algérie. Environ $70 \%$ des rapatriés et des descendants qui ont déjà voté FN éprouvent du ressentiment à l'égard du peuple algérien (Enquête pied-noir 2002). Ce vote s'apparente à une impossibilité de "pardonner " et de dépasser le drame algérien. $85 \%$ des pieds-noirs sondés qui ont déjà voté pour le $\mathrm{FN}$ et $83 \%$ des descendants refusent l'immigration en provenance des pays moins développés (Enquête piednoir 2002). Il peut donc y avoir un vote sur " enjeu " sur ce thème. Par principe, ils sont opposés à la venue d'immigrés nord-africains en France, puisque eux-mêmes ont dû quitter l'Algérie et abandonner là-bas leurs biens et toute une vie, car le peuple algérien voulait son indépendance. La présence en France de personnes d'origine algérienne est une "blessure " qui leur rappelle sans cesse qu'eux ont été, selon leurs dires, "chassés " d'Algérie.
Écoutez, ils nous ont mis dehors de leur pays, ils devraient y rester maintenant, surtout qu'on a laissé là-bas tous nos biens, des infrastruc- tures invraisemblables. On leur a laissé tout ! On est rentré les poches vides. Alors si au moins ils pouvaient se débrouiller et faire fructifier ce qu'on a laissé là-bas et vivre avec, ça serait bien. Il faut être un peu lucide, ils nous ont mis dehors de leur pays, cétait la valise ou le cer- cueil et ici, ils sont accueillis à bras ouverts!» (femme, 65 ans, commer- çante, Constantinoise, Isère).

\begin{abstract}
Les Arabes, en France maintenant, ça devient de plus en plus l'Algérie [...] II y a du ressentiment parce que ce sont des cicatrices qui sont restées ouvertes [...] Pendant les événements, on a perdu des êtres chéris. On nous a mis à la porte [...] On vous disait : « la valise ou le cercueil » (homme, 55 ans, kinésithérapeute, Algérois, Alpes-Maritimes).
\end{abstract}

Dans les entretiens, les Magrébins sont assez souvent perçus comme des Fellaghas, c'est-à-dire comme des personnes qui ont combattu la France pendant la Guerre d'Algérie et qui maintenant vivent sur le sol français et réclament des droits ${ }^{35}$. Des stéréotypes coloniaux sont aussi fréquemment réactivés : les Magrébins sont perçus comme 
fanatiques et violents et foncièrement inassimilables aux valeurs de la République. Des «incompatibilités " cultuelles et culturelles sont souvent évoquées. $80 \%$ des rapatriés et descendants électeurs du FN sondés sont hostiles à ce que les immigrés conservent leurs coutumes et traditions (Enquête pied-noir 2002). En outre, ces piedsnoirs estiment que les Magrébins n'aiment pas la France, profitent abusivement de son système de protection sociale, commettent des délits et veulent imposer leur religion en contestant au quotidien le modèle français de laïcité ${ }^{36}$. Certains, parmi les plus radicaux, sont persuadés que les descendants d'immigrés algériens poursuivent en France la Guerre d'Algérie avec la volonté de "coloniser " à leur tour ce pays ${ }^{37}$. Ils pensent que la France est «menacée » par une immigration maghrébine qu'ils jugent trop importante. Les propositions du FN sur l'insécurité sont également prises en compte, dans la mesure où les pieds-noirs font un amalgame avec l'immigration maghrébine.

Leurs parents viennent de là-bas (Algérie) et c'étaient des anciens du FLN, j'en suis sûr et certain! A part les Harkis38, tout le reste, c'est juste bon à jeter à la mer! (homme, 73 ans, pompier à la retraite, Algérois, Hérault).

Ils sont en train de nous envahir petit à petit. Dieu nous préserve ! [...] Je vois ça d'un mauvais œil! (femme, 65 ans, retraitée de l'Education nationale, Oranienne, Hérault).

On va à une seconde Guerre d'Algérie dans trente ans. La guerre civile existera ici ! [...] Les gouvernants français devraient serrer la vis aux Maghrébins (homme, 36 ans, fils de pieds-noirs, fonctionnaire, Hérault).

Nous, on sait très bien qu'ils ne pourront pas s'intégrer, c'est une race à part, ils ont des coutumes, c'est spécial ! [...] Quand je vois les Arabes en train de voler, de tuer, de casser les bagnoles (femme, 39 ans, fille de pieds-noirs, fonctionnaire, Hérault).

Vous allez dans les prisons, c'est eux qui volent, qui saccagent, il faut dire ce qui est ! [...] Ces gens qui font tant de mal à la France, je les renverrais, puisqu'ils ont un pays! (femme, 66 ans, femme au foyer, Algéroise, Hérault).

36 СомтAт Emmanuelle, « Racial perceptions inherited from the colonial past? From 'indigenous population' to 'immigrant workers': how the 'pieds-noirs' perceive the 'Arabs' from Algeria in postcolonial France ", in Barclay Fiona, Evans Martin et ChopIn Charlotte (dir.), Settler Colonialism in French Algeria, Settler Colonial Studies, Routledge, 2017, pp. 262-282.

37 SAVARÈSE Eric, "Un regard compréhensif sur le traumatisme historique », op. cit., pp. 98-100 et STORA Benjamin, Le transfert d'une mémoire. op. cit., pp. 137-140.

38 Les Harkis sont des supplétifs de l'Armée ayant combattu aux côtés de la France durant la Guerre d'Algérie. 
Chez les autres catégories de Français, le vote en faveur du FN apparait également comme un vote d'opposition aux immigrés ${ }^{39}$. Mais les raisons divergent probablement. Les Français tiennent prioritairement compte de considérations présentes (chômage, insécurité, différence culturelle, religion, terrorisme) tandis que la Guerre d'Algérie est encore déterminante dans le positionnement de leurs compatriotes rapatriés. Les caractéristiques des électeurs frontistes sont d'être hostiles aux immigrés, de ne pas être ouverts aux valeurs universalistes, de revendiquer une société d'ordre et de repli identitaire ${ }^{40}$. II s'agit d'un électorat potentiellement autoritaire.

Des témoignages recueillis auprès de rapatriés après les attentats de Paris en 2015 et de Nice en 2016 indiquent que les actes terroristes ont fait ressurgir des souvenirs de violences commises durant la Guerre d'Algérie. Certains électeurs du FN font l'amalgame entre passé et présent et entre Fellaghas, djihadistes de Isis/Daech et l'ensemble des Musulmans. La peur des attentats a sans doute aussi eu un impact chez les descendants, mais il faudrait comparer leurs réactions face à cette menace (représentations, attitudes) avec celles des autres Français. Marine LE PEN a réorienté son discours anti-immigrés sur l'islamisme qui s'adresse davantage aux nouvelles générations frontistes moins concernées par la période coloniale que par les attentats post 11 septembre ${ }^{41}$.

Les autres propositions du FN (rejet de l'Union européenne, opposition à l'Euro) ne sont pas toujours retenues sauf par certains qui se sentent menacés par la mondialisation.

\section{Une dimension traumatique}

À travers nos différents matériaux empiriques, on observe que les pieds-noirs qui votent pour le FN sont plutôt des personnes qui ont été très profondément marquées par le rapatriement. Dans les entretiens, il s'agit souvent de personnes victimes de violences (physiques ou verbales), d'attentats, ou ayant perdu un proche durant la Guerre d'Algérie. D'autres ont aussi, après le rapatriement, fait une dépression ou ont rencontré plus de difficultés à s'adapter en France.

\footnotetext{
39 Mayer Nonna, "De J-M. Le Pen à M. Le Pen : l'électorat du FN a-t-il changé ? ", in Delwit Pascal (dir.), Le Front national. Mutations de l'extrême droite française, Bruxelles, Editions de l'Université de Bruxelles, 2012 , p. 147.

40 CHICHE Jean et DupoIRIER Elisabeth, «De Chirac à l'UMP: mutations et reconquête », in PERRINEAU Pascal, YSMAL Colette (dir.), Le vote de tous les refus, op. cit., p. 184 et MAYER Nonna, «De J-M. LE PEN à M. LE PEN : l'électorat du FN a-t-il changé ? ", op. cit., p. 147. 
"Après ça a été une haine! On m'a tué une cousine. Elle a laissé trois petits enfants. Quand vous venez de travailler et qu'on vous tire deux balles dans la tête. Après, c'est pas du racisme, c'est de la haine! » (homme, 58 ans, employé communal, Oranais, Isère).

L'enquête quantitative pied-noir 2002 donne des résultats analogues. Les pieds-noirs qui déclarent ne pas avoir surmonté la perte du territoire et qui regrettent leur vie en Algérie ont plus souvent voté pour le FN (Enquête pied-noir 2002). II s'agit aussi des plus nostalgiques du passé colonial. 90\% des pieds-noirs électeurs du FN auraient préféré, avec le recul du temps, le maintien de l'Algérie française (Enquête pied-noir 2002). Ces rapatriés ont également plus souvent que les autres le sentiment que leur situation générale (matérielle et autre) s'est détériorée par rapport à celle qu'ils avaient en Algérie (Enquête pied-noir 2002). Le vote pour le FN résulte donc aussi d'un sentiment de "frustration relative". Les plaies mal cicatrisées de la Guerre d'Algérie agissent encore sur ce vote. Toutefois, les générations les plus meurtries par le rapatriement (celles qui avaient fait leur vie en Algérie et qui ont souvent perdu des biens en quittant ce territoire) tendent à disparaître. Le vote d'une partie des pieds-noirs pour le FN est donc souvent lié au drame de la perte de l'Algérie. Mais comme pour le reste des Français, il est renforcé par la crise de l'emploi et par le sentiment d'insécurité.

La dimension traumatique joue, semble-t-il, moins dans le vote FN chez les descendants qui n'ont pas repris à leur compte les récits familiaux de la Guerre d'Algérie.

\section{Un vote localisé dans le Sud de la France}

Depuis les Européennes de 1984, les scores du FN ont souvent été élevés dans le Midi de la France. Ces régions ont accueilli un contingent important de rapatriés en 1962. Ils y conservent un poids démographique non négligeable et sans doute aussi politique. Selon une estimation de l'IFOP, les rapatriés et les descendants constitueraient en 2012, 12\% du corps électoral en Languedoc-Roussillon, 
15\% en Provence-Alpes-Côte-D'azur, 12\% en Midi-Pyrénées et 11\% en Aquitaine $^{42}$. Le vote $\mathrm{FN}$ dans ces régions a souvent été expliqué par la concentration importante de rapatriés ${ }^{43}$. Qu'en est-il exactement?

Tableau 2 Avoir voté FN en fonction du département de résidence

\begin{tabular}{|l|l|l|l|}
\hline & Isère & Hérault & Alpes-Maritimes \\
\hline Pieds-noirs & $30 \%$ & $45 \%$ & $55 \%$ \\
\hline Descendants & $23 \%$ & $31 \%$ & $39 \%$ \\
\hline
\end{tabular}

Enquête pied-noir 2002 UMR PACTE CNRS

Le vote des pieds-noirs en faveur du FN est, semble-t-il, surtout localisé dans ces régions. Dans les Alpes-Maritimes et l'Hérault, environ la moitié des pieds-noirs sondés déclarent avoir déjà voté pour ce parti (Enquête pied-noir 2002). La part est moindre dans l'Isère où il existe une culture politique de gauche. La propension à voter FN est également plus forte chez les descendants dans le Midi (tableau $n^{\circ} 2$ ). L'enquête préélectorale de 2012 confirme l'existence d'un sur-vote FN dans ce groupe en Provence-Alpes-Côte d'Azur et en LanguedocRoussillon (30\%) en comparaison avec d'autres régions ${ }^{44}$.

Toutefois, les mutations socioéconomiques et politiques dans le Midi, ces cinquante dernières années, ne sont pas étrangères à la percée du FN. Aux premiers rangs desquelles figurent la perte d'influence du port de Marseille avec la fin de l'Empire colonial, la fermeture de chantiers navals dans les années 1980, le taux de chômage élevé, l'arrivée de cadres accaparant les emplois les mieux rémunérés, la perte d'influence du PC dans les milieux populaires, l'affaiblissement de la gauche autrefois dominante dans certaines de ces régions et la montée en puissance de la droite depuis les années 1980, le délitement du lien social, la délinquance, la présence d'immigrés, le nombre important de personnes âgées plus enclines au sentiment d'insécurité, la division de la droite et le climat des affaires ${ }^{45}$. Ces bouleversements n'affectent pas que les rapatriés, mais l'ensemble de la popu-

\footnotetext{
42 IFOP, « Le vote pied-noir : mythe ou réalité », op. cit., p. 2.

43 lbid., p. 6.

$44 \mathrm{lbidem}$.

45 VAN EEUWEN Daniel, «Toulon, Orange, Marignane, le FN au pouvoir : un maléfice méridional ? ", in VIARD Jean (dir.), Aux sources du populisme national. L'urgence de comprendre Toulon, Orange, Marignane, Paris, l'Aube, 1996, pp. 97-131; VAN EEUWEN Daniel et VIARD Jean, Main basse sur la Provence et la Côte d'Azur, La Tour d'Aigues, Editions de l'Aube, 2003, 114 p.; Perrineau Pascal, Le symptôme Le Pen. Radiographie des électeurs du Front national, op. cit., pp. 91-92 et GoMBIN Joël et MAYANCE Pierre, Droites aux urnes en région PACA ! Lélection présidentielle de 2007 en région Provence-Alpes-Côte d'Azur, Paris, L'Harmattan, $2009,300 \mathrm{p}$.
} 
lation locale. Ils ont eu des répercussions sur le vote. Des travaux de science politique ont aussi montré que des descendants d'immigrés espagnols ou italiens établis dans ces régions peuvent être favorables aux thèses extrémistes, car les couches en mal d'ascension sociale sont plus facilement intolérantes ${ }^{46}$, comme les classes moyennes inférieures redoutant d'être déclassées. L'enquête préélectorale de l'IFOP de 2012 montre que, si le niveau de vote pour Marine LE PEN est élevé (30\%) dans la " communauté " pied-noir de Provence-Alpes-Côted'Azur et du Languedoc-Roussillon, il l'est également parmi les $85 \%$ d'électeurs non pieds-noirs de ces régions (21,5\% contre $18 \%$ dans les autres régions). Même sans la présence des pieds-noirs, un sur-vote $\mathrm{FN}$ existerait dans ces deux régions ${ }^{47}$.

En outre, les luttes de la droite $\left(\mathrm{UMP} / \mathrm{LR}^{48}\right)$ et du FN dans le Midi, suite au séisme politique du 21 avril $2002^{49}$, ont contribué à la diffusion des idées extrémistes ${ }^{50}$. Afin de reconquérir des électeurs frontistes, Nicolas SARKOZY et d'autres gaullistes ont repris certains thèmes du discours du FN sur l'immigration et sur les questions identitaires et sécuritaires ${ }^{51}$. Ainsi à la présidentielle de 2007 , selon un sondage de l'IFOP sortie des urnes, 31\% des pieds-noirs auraient voté pour Nicolas SARKOZY, candidat "gaulliste ", tandis que Jean-Marie LE PEN aurait recueilli $18 \%$ de leurs suffrages ${ }^{52}$. À la présidentielle de 2012, Nicolas SARKOZY aurait devancé Marine LE PEN dans l'électorat rapatrié grâce à un positionnement "droitier » et à des signaux envoyés à ce groupe lors de la campagne électorale ${ }^{53}$. Dans cet électorat, la versatilité du vote semble être importante entre ces formations politiques. Les tactiques de «radicalisation » de l'UMP et de «dédiabolisation » du FN ont rendu plus floues les frontières idéologiques de ces partis et entrainé une porosité d'une partie de leurs électorats ${ }^{54}$. Dans le Midi, le durcissement idéologique de la droite a aussi favorisé le rapprochement de responsables d'associations de rapatriés avec les gaullistes. Désormais

46Bon Frédéric et CHEYLAN Jean-Paul, La France qui vote, op. cit., p. 256.

47 SCIENCES PO, CEVIPOF, IFOP, "Le vote pied-noir 50 ans après les accords d'Évian, Élections 2012 ", Les élections sociologiques, 6, 2012, p. 3 et IFOP, "Le vote pied-noir : mythe ou réalité », op. cit., p. 6.

48 L'Union pour un Mouvement populaire (UMP) est un parti de droite gaulliste actif de 2002 à 2015 . II est renommé Les Républicains (LR) en 2015.

49 Le 21 avril 2002, J.-M. LE PEN, candidat du FN, accède au second tour de la présidentielle et doit y affronter J. CHIRAC, candidat de la droite gaulliste.

50 Mayer Nonna, Ces Français qui votent FN, Paris, Flammarion, 1999, pp. 256-257.

51 MaYer Nonna, "Comment N. Sarkozy a rétréci l'électorat Le Pen », Revue française de science politique, 57/3-4, 2007, 429-447 et Perrineau Pascal, La France au Front, op. cit., p. 38 et p. 147.

52 SCIENCES PO, CEVIPOF, IFOP, « Le vote pied-noir 50 ans après les accords d'Évian », op. cit., p. 3.

53 IFOP, "Le vote pied-noir : mythe ou réalité », op. cit., pp. 3-6. 
les revendications mémorielles des associations de rapatriés (commémorations, édification de "lieux de mémoire ", lois mémorielles) sont mieux soutenues par des élus de droite qui rentrent ainsi en concurrence avec des militants du $\mathrm{FN}^{55}$.

\section{Un vote contestataire}

Le vote de certains pieds-noirs en faveur du FN revêt également une "dimension contestataire ". Le même phénomène est observé dans d'autres catégories de Français ${ }^{56}$. Le vote contestataire est l'expression d'une exaspération face à la situation politique et sociale de la France et un "avertissement " envoyé à la classe politique. Le FN a su exploiter le désenchantement politique de ces trente dernières années qui s'est progressivement transformé en ressentiment et en colère, exprimés dans la montée de l'abstention et dans le vote pour les partis "hors système " $^{57}$. Chez les rapatriés, le vote contestataire s'inscrit dans ce contexte, mais il est aussi souvent lié à la Guerre d'Algérie. Passé et présent sont chez nombre d'entre eux entremêlés. Le FN a su attirer une partie de ceux que le vent de l'histoire et la fin des colonies avaient "balayés " et marginalisés et qui ne se sentaient plus représentés par les partis traditionnels. Certains estiment que les hommes politiques les ont "trahis » en Algérie et qu'aujourd'hui ils se soucient peu des problèmes des Français, intéressés seulement par leur carrière politique. Le vote en faveur du FN vise alors à renvoyer "dos à dos " les partis républicains qui gouvernent la France depuis 50 ans. Dans sa propagande, le FN a instrumentalisé la désillusion à l'égard du clivage gauche-droite (dénonciation de "l'UMPS ${ }^{58}$ ") pour se poser en alternative ${ }^{59}$.

Dans les entretiens réalisés en 2000 , des pieds-noirs faisaient plus facilement entendre leur mécontentement dans les urnes aux élections nationales. D'autres logiques étaient à l'œuvre aux échéances locales (liens clientélistes, fidélité à une personnalité politique) qui les faisaient soutenir des candidats de partis traditionnels. Des piedsnoirs votaient aussi pour le FN au premier tour de la présidentielle et se réalignaient au second tour sur les partis de droite (UMP/LR) ou du centre (Modem, UDI). Aux dernières élections, certains franchissent

\footnotetext{
55 COMTAт Emmanuelle, «Du vote des pieds-noirs aux politiques mémorielles à l'égard des rapatriés : étude du lien entre une opinion publique catégorielle et l'Action publique », Pôle Sud, 45, 2016, pp.121-145. 56 Perrineau Pascal, "Le Front national d'une élection à l'autre », Regards sur l'actualité, n¹61, 1990, p. 24. 57 Perrineau Pascal, La France au Front, op. cit., pp. 159-170.

58 L'UMPS désigne l'UMP et le PS pour indiquer qu'il n'y a aucune différence de politique entre ces partis. 59 Perrineau Pascal, La France au Front, op. cit., pp. 165-166.
} 
le pas d'un vote $\mathrm{FN}$ au plan local, considérant qu'il s'agit "d'un parti comme un autre " à qui il faut donner sa chance, car les autres formations politiques ont échoué. Les élections municipales de 2014 ont permis au FN ou aux listes apparentées de remporter une dizaine de communes de plus de 9000 habitants dans le Midi ou dans le Nord et l'Est de la France ${ }^{60}$. Désormais le $\mathrm{FN}$ compte 358 conseillers régionaux et 62 conseillers départementaux élus en 2015 et 8 députés élus en 2017.

\section{Caractéristiques sociodémographiques des rapatriés électeurs du FN}

L'enquête pied-noir 2002 réalisée il y a seize ans dressait une sociologie du vote des pieds-noirs. Son objectif était d'observer si les variables sociodémographiques (âge, niveau de diplôme, catégorie socioprofessionnelle, sexe, pratique religieuse) explicatives du vote FN chez l'ensemble des Français continuaient d'être opérantes dans ce groupe marqué par une histoire singulière et traumatique. II n'existe pas d'enquête plus récente prenant en compte ces dimensions sociologiques. Celles de l'IFOP en 2012 s'intéressent davantage au poids du vote $\mathrm{FN}$ selon sa localisation géographique qu'aux déterminants sociologiques des positionnements électoraux. II n'est donc pas possible de déterminer si la stratégie de "dédiabolisation » entreprise par les nouveaux dirigeants du FN modifie la sociologie de cet électorat chez les rapatriés et leurs descendants. Les résultats présentés ici sont issus de l'enquête de 2002. 
Tableau n³ Avoir déjà voté FN en 2002 selon la profession, le niveau de diplôme et la pratique religieuse

\begin{tabular}{|l|l|l|}
\hline & Pieds-noirs & Descendants \\
\hline À son compte & $41 \%$ & $43 \%$ \\
$\begin{array}{l}\text { Professions intellectuelles } \\
\text { supérieures }\end{array}$ & $45 \%$ & $11 \%$ \\
Professions intermédiaires & $44 \%$ & $21 \%$ \\
Employés & $41 \%$ & $35 \%$ \\
Ouvriers & $38 \%$ & $41 \%$ \\
\hline Inférieur au bac & $43 \%$ & $38 \%$ \\
Supérieur ou égal au bac & $46 \%$ & $25 \%$ \\
\hline $\begin{array}{l}\text { Catholiques pratiquants régu- } \\
\text { liers }\end{array}$ & $42 \%$ & $40 \%$ \\
Catholiques pratiquants irré- & $56 \%$ & $33 \%$ \\
guliers & $48 \%$ & $34 \%$ \\
Catholiques non pratiquants & $4 \%$ & $0 \%$ \\
Juifs & $30 \%$ & $23 \%$ \\
Sans religion & &
\end{tabular}

Enquête pied-noir 2002 UMR PACTE CNRS

La catégorie socioprofessionnelle et le niveau d'éducation n'ont pas d'incidence sur le fait de voter pour le FN chez les rapatriés (tableau n`3). Quelle que soit leur milieu social, ils ont été collectivement confrontés au traumatisme de la perte de l'Algérie, ce qui expliquerait ce positionnement atypique. En revanche, chez l'ensemble des Français, les cadres et les professions intermédiaires sont les catégories les plus réticentes à voter pour le FN. Plus exposés aux changements de modes de production et à la concurrence, les ouvriers, les commerçants et artisans votent, quant à eux, plus pour l'extrême droite ${ }^{61}$. Le FN progresse chez les ouvriers, les employés et les chômeurs ${ }^{62}$. La faiblesse du niveau d'éducation conduit aussi à mieux accepter la vision simpliste de la société que propose le FN, à réclamer une société d'ordre et à être plus autoritaire ${ }^{63}$. Les enfants de pieds-noirs qui votent FN rejoignent pour leur part davantage le « pro-

61 MAYER Nonna, Ces Français qui votent FN, op. cit., pp. 61-74 et pp. 85-97 ; CAUTRÈs Bruno et MAYER Nonna, "Les métamorphoses du vote de classe ", in CAUTRĖs Bruno et MAYER Nonna (dir.), Le nouveau désordre électoral, Paris, Presses de Sciences Po, 2004, pp. 150-152 et PerRINEAU Pascal, Le symptôme LE PEN. Radiographie des électeurs du Front national, op. cit., pp. 107-111.

62 Perrineau Pascal, La France au Front, op. cit., pp. 39-40. 
fil classique " des électeurs français qui soutiennent ce parti. La crise de l'emploi a un impact plus important dans cette génération moins exposée au choc de la décolonisation et en âge d'être en activité.

Par ailleurs, le sexe a une influence forte sur ce vote en 2002. Les hommes votent davantage pour le $\mathrm{FN}$ que les femmes ( $50 \%$ contre $36 \%$ ) (Enquête pied-noir 2002). Cette différence, selon le genre, rejoint alors les conclusions des études explicatives du vote FN. Les femmes sont à l'époque moins attirées par les partis qu'elles jugent "excessifs » et par la personnalité de Jean-Marie LE PEN ${ }^{64}$. Chez les enfants de pieds-noirs, l'écart est en revanche moindre entre les sexes. Marine LE PEN a en partie levé ce verrou selon le genre et les enquêtes récentes révèlent que les femmes votent davantage qu'avant pour ce parti perçu comme moins autoritaire, mais à un niveau moindre que les hommes ${ }^{65}$.

Tableau $n^{\circ} 4$ Vote $\mathrm{FN}$ en fonction de l'âge des individus en 2002

\begin{tabular}{|l|l|l|l|l|}
\hline & $\begin{array}{c}\text { Nés en 1903- } \\
1922\end{array}$ & $\begin{array}{c}\text { Nés en 1923- } \\
1946\end{array}$ & Nés en 1947-1952 & $\begin{array}{l}\text { Nés en 1953- } \\
1962\end{array}$ \\
\hline A voté FN & $31 \%$ & $46 \%$ & $34 \%$ & $22 \%$ \\
\hline
\end{tabular}

Enquête pied-noir 2002 UMR PACTE CNRS

L'âge joue également (tableau n4). II s'agit davantage d'un effet de génération. Les rapatriés sont plus nombreux à avoir déjà voté $F N$ que leurs descendants. Seuls les plus âgés de l'échantillon (les plus de 80 ans en 2002) ont un peu moins souvent voté pour le FN. Mais les effectifs plus restreints de cette catégorie d'âge du fait du vieillissement introduisent un biais d'enquête qui ne permet pas de tirer de conclusions ${ }^{66}$. L'enquête préélectorale de l'IFOP de 2012 complète ces résultats en montrant qu'à âge égal, les rapatriés ont plus souvent voté pour Marine LE PEN que leurs compatriotes français. Dans cette génération, la Guerre d'Algérie continue d'exercer une incidence sur les comportements électoraux. Le vote pied-noir conserve ainsi des spécificités. En revanche, elles s'estompent chez leurs descendants, puisque le vote des personnes ayant une ascendance pied-noir est très proche des électeurs sans ascendance de ce type. Le processus de réalignement électoral des enfants de pieds-noirs s'est effectué dès

64 Op. cit., pp. 117-131.

65 Perrineau Pascal, La France au Front, op. cit., p. 40 et Mayer Nonna, « De J-M. Le Pen à M. Le PEN : l'électorat du FN a-t-il changé? ", op. cit., p. 150.

66 Certains, dans cette génération mobilisée pour combattre le nazisme après le débarquement angloaméricain de 1942 en Algérie, peuvent avoir des réticences à voter FN. 
la seconde génération. Toutefois, l'enquête de l'IFOP de 2012 montre que ces derniers ont désormais l'intention de voter pour le FN dans les mêmes proportions que les rapatriés ${ }^{67}$. L'explication réside dans le fait que, dans la population non pied-noir qui a servi de base de comparaison, les 24-34 ans et les 35-49 ans sont des tranches d'âges qui votent assez fortement pour le $\mathrm{FN}^{68}$. Marine LE PEN a su rajeunir l'électorat de ce parti en s'adressant aux plus exposés au chômage ${ }^{69}$. Les enfants de pieds-noirs sont donc ici très semblables à leurs compatriotes de même génération et les causes de ce vote sont à trouver dans les problèmes actuels de l'emploi et de la précarisation liés à la mondialisation que dans les effets à long terme du traumatisme du rapatriement. S'ils votent presque autant pour le FN, les descendants sont néanmoins plus nombreux que leurs ainés à soutenir des candidats de gauche au détriment de ceux de la droite " gaulliste $»^{70}$. Les transferts de voix semblent s'opérer essentiellement entre l'UMP/LR et le $\mathrm{FN}$, ce qui montre que le $\mathrm{FN}$ est considéré par certains électeurs comme un parti de droite comme un autre.

La religion a aussi en 2002 une incidence sur le vote $\mathrm{FN}$ dans ce groupe des rapatriés (tableau n`3). Comme chez l'ensemble des Français, le vote pour le FN est surtout le fait des catholiques non pratiquants ou des pratiquants irréguliers ${ }^{71}$. Dès les premiers succès électoraux de ce parti, l'Église catholique a clairement condamné les idées du FN considérées comme incompatibles avec le message des Évangiles. Toutefois, dans la génération des descendants la pratique religieuse n'empêche pas complètement de voter FN. Par ailleurs, les pieds-noirs de confession juive et leurs enfants ainsi que les sans religion votent en 2002 nettement moins pour le FN que les catholiques, quel que soit leur degré de pratique religieuse. Ils sont globalement hostiles à l'extrême droite qui, dans l'histoire, a persécuté les juifs ${ }^{72}$.

«Il faut dire que je suis juif, que par définition, l'extrême droite, c'est le monstre!» (homme, 52 ans, restaurateur, Oranien, Alpes-Maritimes).

Néanmoins, des enquêtes récentes révèlent que l'accession de Marine LE PEN à la tête du FN et sa volonté de rompre avec les ambigüités de son père sur l'antisémitisme auraient fait progresser le

\footnotetext{
67 À la présidentielle de $2012,21,5 \%$ des pieds-noirs ont l'intention de voter FN contre $20 \%$ de leurs descendants et $18 \%$ des personnes sans ascendance pied-noir ; IFOP, «L Le vote pied-noir : mythe ou réalité », op. cit., p. 6 .

68 IFOP, «L Le vote pied-noir : mythe ou réalité », op. cit., pp. 5-6.

69 MAYer Nonna, «De J-M. Le Pen à M. Le PeN : l'électorat du FN a-t-il changé? », op. cit., p. 150.

70 IFOP, «Le vote pied-noir : mythe ou réalité », op. cit., p. 6.

71 Perrineau Pascal, Le symptôme Le Pen. Radiographie des électeurs du Front national, op. cit., pp. 111-113.

72 StRUdel Sylvie, Votes juifs : itinéraires migratoires, religieux et politiques, Paris, Presses de Sciences Po, 1996.
} 
vote FN dans l'électorat de confession juive traumatisé par le terrorisme islamiste qui a visé en France cette communauté depuis 2012. Le vote $\mathrm{FN}$ demeurerait cependant fortement minoritaire ${ }^{73}$.

\section{Conclusion}

Le passé traumatique continue d'exercer une influence sur l'orientation politique des rapatriés. Pour autant il n'a pas eu pour effet d'orienter les rapatriés vers un parti en particulier. Leur vote est très divers. II l'était déjà en Algérie avant 1962 et l'est encore aujourd'hui. Toutefois, les rapatriés sont majoritairement à droite. Un sur-vote en faveur du FN les distingue aussi de leurs compatriotes Français probablement en raison du traumatisme du rapatriement. Les rapatriés qui votent FN n'ont pas "pardonné " la perte de l'Algérie et n'acceptent pas la présence en France de populations magrébines qui leur rappellent cette défaite et cette blessure. Les variables sociodémographiques ordinairement explicatives du vote fonctionnent moins bien dans ce groupe. Par contre, les descendants se réfèrent moins au passé dans le façonnement de leur opinion politique aujourd'hui. Le drame vécu par les pieds-noirs ne les concerne pas personnellement. Ils conservent moins de spécificités que leurs aînés. Des mécanismes de recomposition des opinions sont à l'œuvre dans ce groupe. Les descendants qui votent $\mathrm{FN}$ ressemblent davantage à leurs compatriotes de même génération électeurs de ce parti. Leurs motivations à voter pour le FN apparaissent similaires et résultent d'inquiétudes actuelles. Les variables sociodémographiques redeviennent explicatives du vote FN dans cette génération. Il est donc hasardeux de parler de «traumatisme historique " au sens de Paul Bols, dans la mesure où les effets politiques de ce traumatisme ne semblent pas se pérenniser d'une génération à l'autre. 